\title{
Lateral Summation and Inhibition in the Human Retina
}

\author{
By
}

\section{Tadashi Aizawa and Shin'itiro Katayama}

(相 沢 匡) (片山 新一郎)

From the Physiological Laboratory of Prof. K. Motokawa, Tohoku University, Sendai

(Received for publication, January 24, 1956)

\section{INTRODUCTION}

There is much evidence ${ }^{1-4)}$ which tends to show that spatial summation takes place in the retina. For instance, Adrian and Matthews ${ }^{5 / 6}$ ) have demonstrated the interaction of retinal neurones by recording the impulses in the optic nerve of the conger eel. When four points on the retina were illuminated simultaneously, the reaction time of the optic nerve discharge was shorter than when any one of the points was illuminated alone. This effect was similar to the one following an increase in the intensity of the stimulus.

Later, Granit ${ }^{78)}$ determined flicker fusion frequencies for similar four circular test spots together and for each of the spots alone and found spatial summation effects in the human retina. Furthermore, spatial summation in the human retina was studied by Motokawa ${ }^{9)}$ with increases of electrical excitability by illumination as an index. He has shown that increases of electrical excitability after exposure to five circular spots as indicated in Fig. 1 (A) or to four circular spots, was always higher than those caused by exposure to any one of them alone. The closer the five spots together, the greater the value of increases of electrical excitability. Summation was weaker as the distance separating spots increased, and no indication of interaction was seen at a separation of about $6^{\circ}$ in visual angle. Motokawa did not work with greater separations, for he took for granted that no interaction would take place at a greater separation. It was, however, found by chance that the absence of any summation at a separation of about $6^{\circ}$ is a phenomenon of transition from summation to inhibition. In the present investigation this relation was established by exploring a much greater extent of retinal areas with two different methods. 


\section{EXPERIMENTAL}

\section{Methods}

Two different methods were used. One is the same as that used in Motokawa's experiment, that is, measurements of electrical excitability of the eye, the other is a binocular brightness match method similar to that used by Schouten \& Ornstein. ${ }^{10}$ )

1) Measurements of electrical excitability of the eye after exposure to light $(\zeta$-method)

The details of the procedure involved are described in Motokawa'sil paper, so that they may be omitted in this report. Experiments on the human eye were carried out in a dark room after preliminary dark adaptation of about 20 minutes. Following an illumination, the electrical sensitivity of the eye is measured with electrical phosphenes as an index. Percentage increases in electrical sensitivity after exposure to light are denoted by $\zeta$. According to Motokawa ${ }^{1213)}$ the $\zeta$-value determined 2 seconds after a white light stimulus lasting 2 seconds represents the magnitude of the cone process, and the $\zeta$-value determined 4.5 seconds after a flash of light represents the magnitude of the rod process.

Such arrangements of test patches as indicated in Fig. 1 (A) and

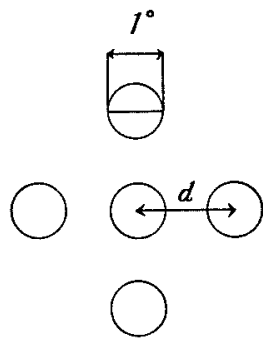

$A$

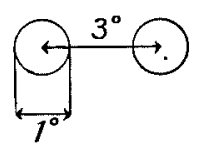

B

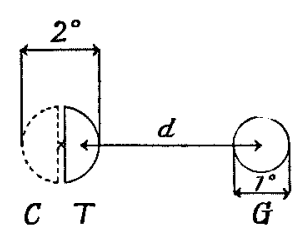

C.

Fig. 1. Arrangement of test patch.

(B) were used in these experiments. Let the value of $\zeta$ for the central spot alone be $\zeta_{1}$, and that for all spots together be $\zeta_{2}$, then the ratio $\phi=$ $\zeta_{2} / \zeta_{1}$ serves as an index of interaction; it indicates summation when $\phi>1$, and inhibition when $\phi<1$.

2) Method of binocular brightness match

The aspect of the apparatus used is seen in Fig. 2. Light sources $\mathrm{A}, \mathrm{B}$ and $\mathrm{D}$ are separated from one another with light-tight walls. At distance of $30 \mathrm{~cm}$. from the observer's eye, there are three independent 


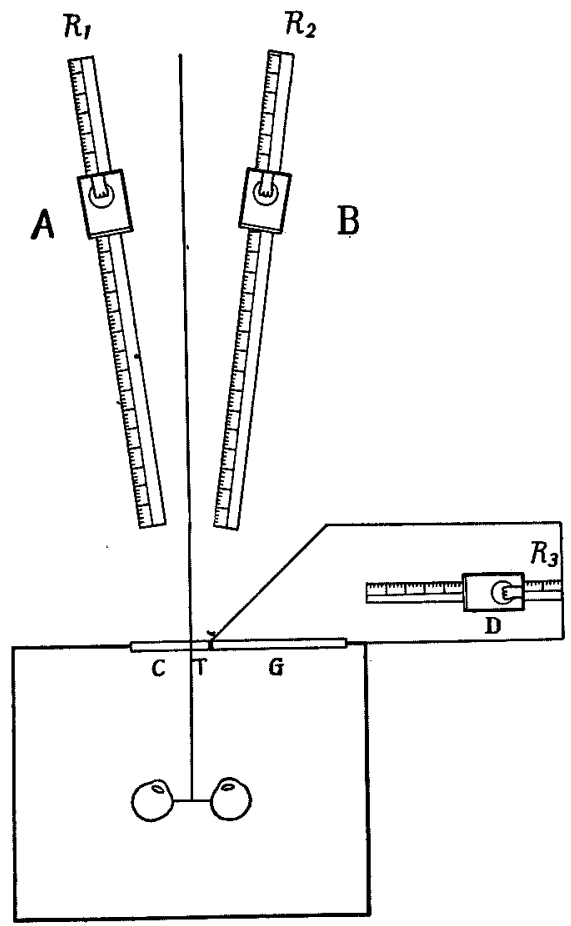

Fig. 2. Apparatus for binocular brightness match method.

A : Light source for comparison patch.

B : Light source for test patch.

C : Comparison patch. D : Light source for glare patch.

$G$ : Glare patch. $R_{1}, R_{2}$ and $R_{3}$ : Optical benches.

$\mathrm{T}:$ Test patch.

patches $\mathrm{C}, \mathrm{T}$ and $\mathrm{G}$ in a perpendicular screen. $\mathrm{C}$ and $\mathrm{T}$ are semicircular patches of $2^{\circ}$ in diameter cut from a sheet of black paper covering the perpendicular screen of ground glass, and placed in such a way that the left eye sees only $\mathrm{C}$ and the right one only $\mathrm{T}$. The patch $\mathrm{C}$ is illuminated by the light source $\mathrm{A}$ of a fixed intensity and serves as a control patch. The test patch $T$ is illuminated by the light source $B$ which is movable along an optical bench $R_{2}$. The glare patch $G$ is so arranged that the physical intensity of illumination at the test patch $T$ is not influenced by G. The glare patch $\mathrm{G}$ is an opening of $1^{\circ}$ cut from a sheet of black paper covering a ground glass screen, which is illuminated with the glare source $D$ movable along $R_{3}$. The distance between $T$ and $G$ or the glare angle $\mathrm{d}$ can be varied from $2^{\circ}$ to $20^{\circ}$.

The experimental room is dimly illuminated, and the eyes of the observer are adapted to this illumination. The experimenter adjusts 
the intensity of $\mathrm{T}$ roughly till an approximate match between $\mathrm{C}$ and $\mathrm{T}$ is secured, and then an exacter match is obtained by shifting B slightly forward or backward. In the matching a special difficulty arises with inexperienced observers because of the wandering convergence of the eye axes. This manifests itself in a seemingly different relative position of the two objects in the field of view. After some experience, however, this convergence comes under conscious control so that the two halves of the photometric field can be made to join exactly. Care is taken in the routine measurements always to fixate the exact center of the circular field formed by the two objects. After the objects are matched, the right eye is illuminated with the glare light. So the test field is suddenly seen darker than the comparison field. During exposure, light source B is moved forward until again a match is obtained. All readings are taken 5 seconds after the beginning of exposure. If the intensity of $T$ necessary to match $\mathrm{G}$ with and without the glaring illumination be $\mathrm{E}_{2}$ and $\mathrm{E}_{1}$, the ratio $\rho=\mathrm{E}_{1} / \mathrm{E}_{2}$ will serve as an index of interaction; the index greater than unity means summation, and that smaller than unity inhibition.

Results

\section{1) Summation and inhibition as a function of distance between stumuli}

Such an arrangement of test patches as indicated in Fig. 1 (A) was used in this series of experiment. Each spot had a diameter $1^{\circ}$ visual angle, and the distance $\mathbf{d}$ between the central and peripheral spots was varied between $1.5^{\circ}$ and $11^{\circ}$. The central spot was always centrally fixated. The intensities of illumination were 50, 500 and $5000 \mathrm{lux}$, and the period of exposure 2 seconds. Electrical sensitivity was determined 2 seconds after the end of illumination, and values of $\zeta$ were determined for the central spot alone and for all five together to obtain $\phi$. The data are indicated in the upper diagram of Fig. 3 as $\phi$-d curves. The ratio $\phi$ is greater than unity when the distance is small (summation), but decreases with increasing distances to become unity at a certain distance which is smaller for higher intensities. With further increases of $d$, the ratio becomes less than unity (inhibition). The degree of inhibition is greatest at an intermediate distance ranging $8^{\circ}$ to $11^{\circ}$. No indication of interaction could, however, be found at a greater distance such as over $15^{\circ}$.

Similar experiments were carried out in the periphery of the retina, the center of the five spots being placed either at $10^{\circ}$ or $20^{\circ}$ from the fovea. The intensity of illumination was 500 lux. The results are shown in the lower diagram of Fig. 3. In these peripheral regions of the retina only summation is seen, but no inhibition at all. If the inhibition were to 

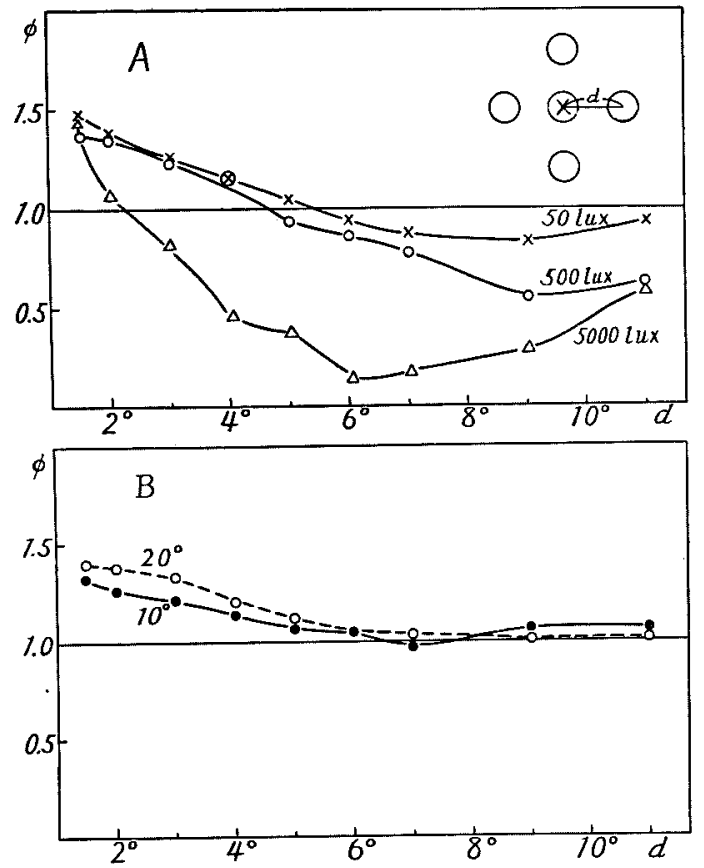

Fig. 3. Relation between index of interaction $\phi$ and distance between central and peripheral spots d. Upper diagram refers to central fixation of central spot, and lower diagram to peripheral fixation of it. Continuous and broken curves were obtained with central spot at $10^{\circ}$ and $20^{\circ}$ from fovea respectively. Summation $(\phi>1)$, inhibition $(\phi<1)$. Intensity of illumination was 500 lux.

take place at a certain distance between the central spot and the surrounding spots, it would be expected to take place in the latter experiment as well, because the configuration of the patch used was the same in both experiments. The central spot was situated at the fovea in the first experiment, but at $10^{\circ}$ or $20^{\circ}$ from the fovea in the second. If the $\zeta_{1}$-value for the central spot alone were definitely lower for some reasons at the periphery than at the fovea, the ratio $\zeta_{2} / \zeta_{1}$ would always be greater than unity. The summation observed in the second experiment might be an apparent one due to such a simple reason. In reality, however, the $\zeta_{\mathbf{1}^{-}}$ values obtained were 57,55 and 50 at the fovea, $10^{\circ}$ and $20^{\circ}$ respectively; the difference is obviously too small to account for the great difference observed between the two experiments. The highest value of $\phi$ was 1.5 and the lowest 0.54 in the first experiment, whereas the highest and the lowest were 1.4 and 0.98 in the second. Thus, the amplitude of $\phi$ is far greater in the first experiment than in the second. This difference cannot 
be accounted for merely in terms of shifts of the reference values $\zeta_{1}$.

The inhibition was very conspicuous when four spots fell upon peripheral areas $8^{\circ}-11^{\circ}$ from the fovea in the first experiment. The question now arises as to whether illumination of these parafoveal regions causes especially strong inhibition. The following experiment was performed to answer this question.

2) Inhibition in parafoveal regions of the retina

The patch used consisted of two spots $1^{\circ}$ in visual angle with centers $3^{\circ}$ apart. This patch was presented at various positions in the temporal horizontal meridian. The ratio of $\zeta_{2}$ for two spots together to $\zeta_{1}$ for the left spot alone was denoted by $\phi$. The data are shown in Fig. 4 where

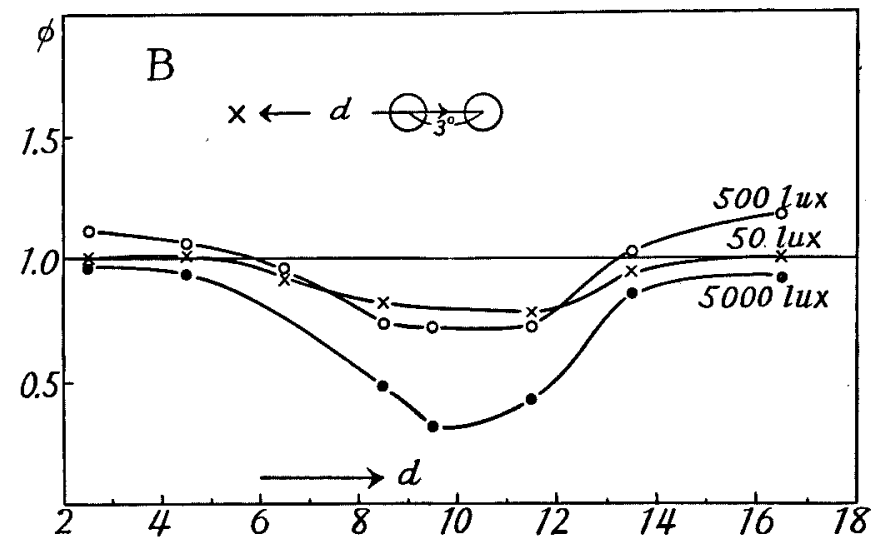

Fig. 4. Regional difference of inhibition determined by two spots method. Abscissae: Retinal position of center of gravity of two spots. Fixation point is marked by cross.

the location of the patch is indicated by the position of the center of gravity of the two spots. A slight degree of summation can be observed near the fovea as well as far in the periphery, but elsewhere inhibition; especially it is strong in the parafoveal region $8^{\circ}-11^{\circ}$ from the fovea.

Similar measurements were carried out in other 7 meridians, the intensity of illumination being fixed at 500 lux. The data obtained are represented by a topogram in Fig. 5. In this figure, the cross hatched area and the dotted area represent a stronger and weaker inhibitory area respectively and the inner and outer blank areas summatory areas.

It should be noted that the stronger inhibitory area covers a zone whose inner and outer diameters $16^{\circ}$ and $22^{\circ}$. When two spots fall within this inhibitory area, their effect in terms of $\zeta$ is definitely smaller than the effect of either of them. In the five spots experiments we have observed that the effect of the five spots together was much smaller than that of 


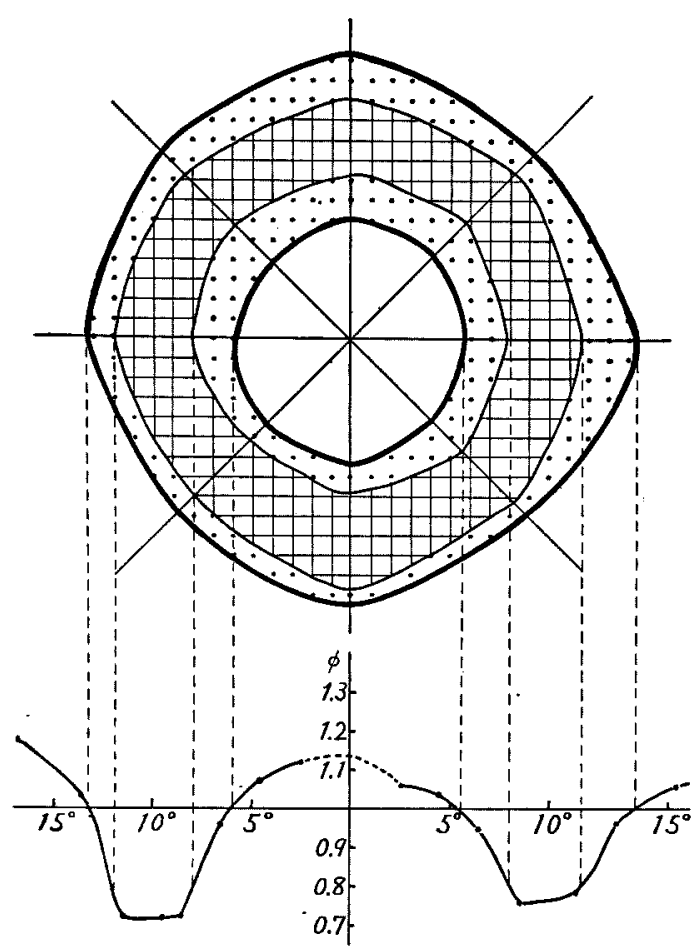

Fig. 5. Topogram of spatial summation and inhibition on the retina. Areas of strong and weak inhibition are cross hatched and dotted respectively. Area of summation is blank.

the central spot alone when the peripheral four spots fell upon the same inhibitory zone. From these facts it is likely that some inhibitory action is initiated within this inhibitory zone when illuminated, and that excitation either within the area or outside the area must be depressed by the inhibitory action thus initiated.

So far the values of $\zeta$ was measured 2 seconds after presentation of white light for a period of 2 seconds. This $\zeta$-value represents the magnitude of the cone process. According to Motokawa, ${ }^{12) 13}$ ) $\zeta$-values determined 4.5 seconds from the onset of an illumination represent the magnitude of the rod process.

In the following experiment summation and inhibition of the rod process were studied with arrangement of test patches shown in Fig. 1 (B). The pre-illumination was made with a flash of white light lasting 40 msec. The results are reproduced in Table I. All values of $\phi$ are greater than unity. In other words, there is no indication of inhibition at all, even at such a high intensity of illumination as 300 lux. It is to 
be noted that no inhibition takes place of the rod process even within the inhibitory zone mentioned above. Therefore we may mention of inhibition only with regard to the cone process.

\section{TABLE I}

Summation in Various Regions of the Retina

(two spots experiment)

Distance between two spots: $3^{\circ}$ (Fig. 1 (B)). Preliminary illumination is a flash of white light of $40 \mathrm{msec} . \zeta_{1}: \zeta$ for left spot alone. $\zeta_{2}: \zeta$ for two spots together. $\phi=\zeta_{2} / \zeta_{1}$

\begin{tabular}{|c|c|c|c|c|c|c|c|c|c|}
\hline \multirow{2}{*}{$\begin{array}{l}\text { Location } \\
\text { (degrees } \\
\text { from center) }\end{array}$} & \multicolumn{3}{|c|}{$1 \operatorname{lux}$} & \multicolumn{3}{|c|}{30 lux } & \multicolumn{3}{|c|}{$300 \operatorname{lux}$} \\
\hline & $\zeta_{1}$ & $\zeta_{2}$ & $\phi$ & $\zeta_{1}$ & $\zeta_{2}$ & $\phi$ & $\zeta_{1}$ & $\zeta_{2}$ & $\phi$ \\
\hline 5 & 12.5 & 14.5 & 1.12 & 21.5 & 38 & 1.77 & 19 & 25 & 1.32 \\
\hline 7.5 & 14.5 & 18.5 & 1.28 & 20 & 36 & 1.80 & 23 & 29 & 1.26 \\
\hline 10 & 15.5 & 22 & 1.41 & 25 & 43 & 1.72 & 23 & 28 & 1.21 \\
\hline 13 & 15.5 & 19.5 & 1.26 & 26.5 & 43 & 1.62 & 22 & 28 & 1.32 \\
\hline 17 & 19.5 & 23 & 1.18 & 30 & 43 & 1.43 & 23 & 31 & 1.34 \\
\hline
\end{tabular}

3) Quantitative measurements of the sensitivity level during exposure

Method of binocular brightness match was used in this series of experiment. Since the experimental apparatus and procedure are already stated in an item of the experimental methods, there seems to be no need to elaborate it again. The illuminations of the test patch $T$ before and during exposure to glare light $\mathrm{G}$ are denoted by $\mathrm{E}_{1}$ and $\mathrm{E}_{2}$ respectively and the ratio $\mathrm{E}_{1} / \mathrm{E}_{2}$ is expressed by $\rho$. The ratio $\rho$ was measured as a function of the intensity of the glare light and the glare angle d. Fig. 6 represents a series of such measurements: Each point gives the mean value of ten readings. The intensity of the glare light was varied between 1.3 and 1000 lux by moving light source $D$ along $R_{\mathbf{3}}$. It is seen that the glare effect (a decrease of $\rho$ below 1) decreases rapidly with increasing glare angles, and increases as the intensity of illumination rises. It should, however, be noted that there is an inflection on each $\rho$-glare angle curve except the one for the lowest intensity (1.3 lux). This inflection is seen over $7^{\circ}-11^{\circ}$ glare angles, a retinal region to be identified with the inhibitory zone mentioned above.

In the following experiment the glare angle was fixed at $3^{\circ}$, and the point of fixation was moved along the vertical meridian so as to make brightness matches at various regions of the retina. At each retinal region hundred readings were taken. The data are represented as a function of vertical distances from the fovea in Fig. 7. The intensity of 


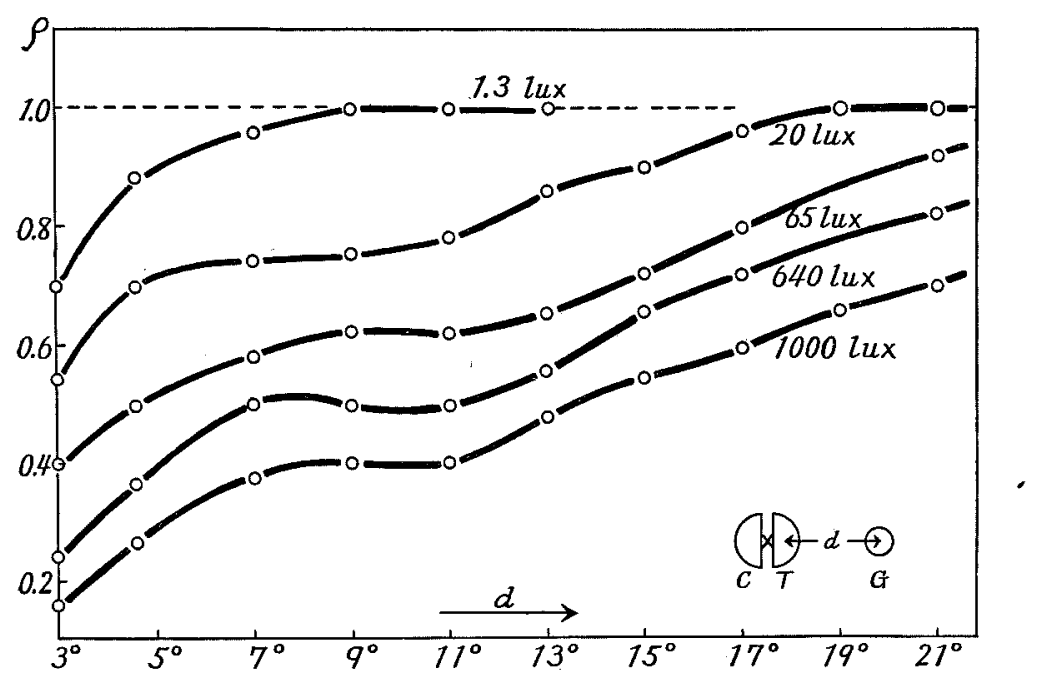

Fig. 6. Glare effect as function of glare angles (brightness match method). Summation $(\rho>1)$, inhibition $(\rho<1)$.

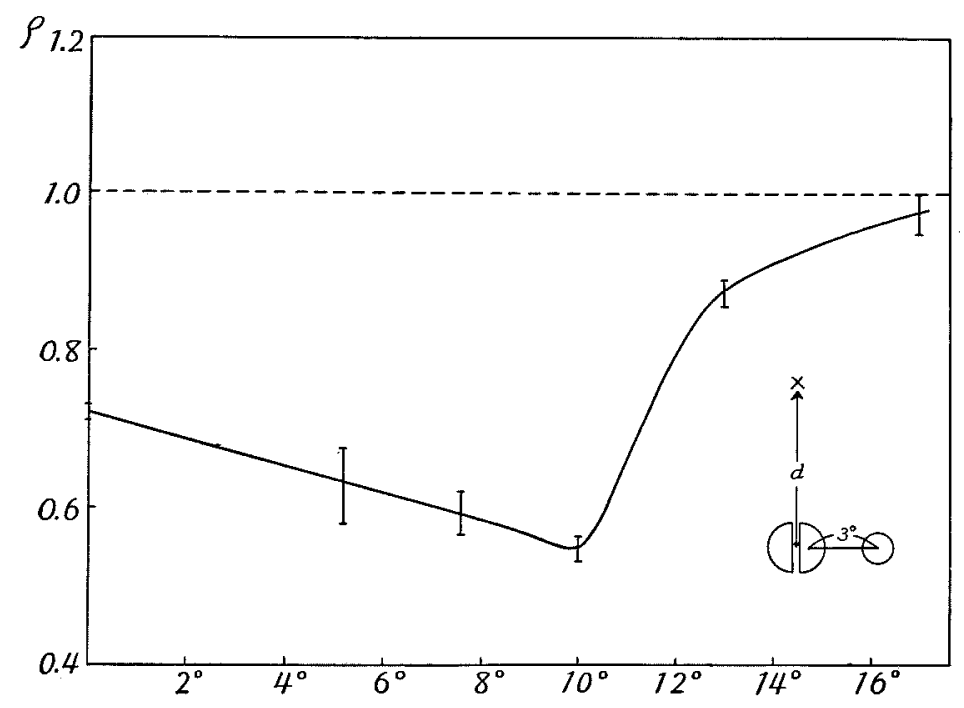

Fig. 7. Regional difference of glare effect.

the glare light was always 1000 lux. The vertical lines on the curve indicate the standard deviation of measurements at each point. The glare effect is strongest in the retinal region $8^{\circ}-10^{\circ}$ from the center in agreement with the result obtained with the $\zeta$-method.

Finaly, two supplementary experiments were carried out so as to 
investigate separately glare effects upon scotopic and photopic systems. In one experiment all conditions favorable for selective excitation of the rod system were adopted, e.g. 1. complete dark adaptation, 2. blue working light and 3. use of light of low illumination. The glare patch $\mathrm{G}$ was presented on the same side as the control patch $\mathrm{C}$, the illumination of these patches being 0.2 lux. The data are represented in the upper diagram of Fig. 8. Vertical lines on the curve represent standard deviations of 100 readings. In presentation of $G$ and $G$ patches together $G$ looks brighter than in presentation of $\mathrm{C}$ alone, that is, there is summation, but not at all inhibition in accordance with the result for the rod process obtained by the $\zeta$-method.

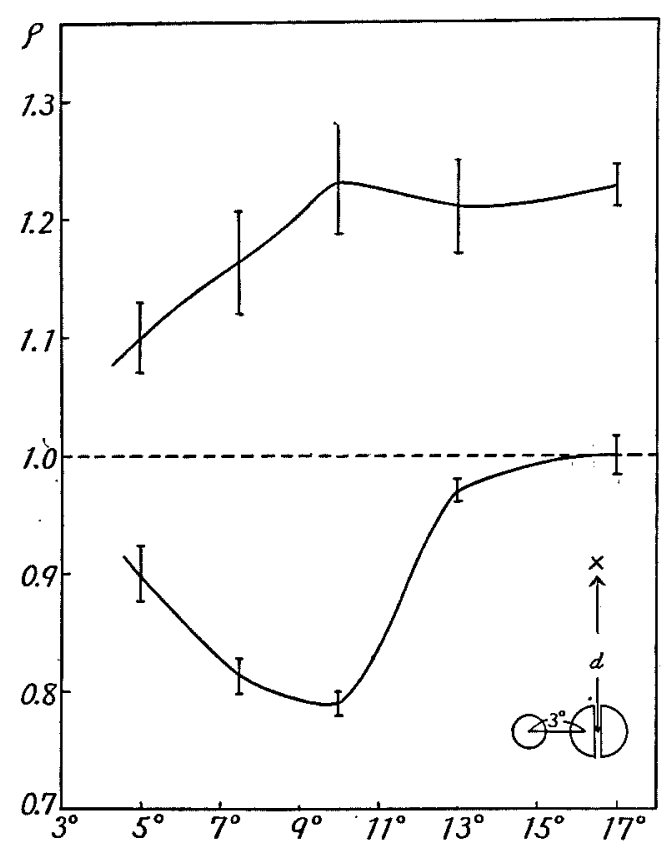

Fig. 8. Variation of brightness caused by simultaneous illumination of a neighboring area in scotopic (upper diagram) and photopic (lower diagram).

Similar measurements were performed with red light of higher intensity, namely 20 lux to excite selectively the cone system. The data are shown in the lower diagram of Fig. 8, which shows a definite inhibition over a parafoveal region in contrast to the data obtained above for the rod system.

\section{Discussion}

Kuffler ${ }^{14115)}$ has demonstrated the interaction of different regions 
within a single receptive field by simultaneous excitation with two small beams of light. In his papers it was shown that the receptive field was $1-4 \mathrm{~mm}$. in diameter and that functionally the center and the surrounding regions of the receptive field are opposed, the one tending to suppress the other. Therefore it is possible that the inhibition caused by illumination of the parafoveal inhibitory zone in our experiments is related to the peripheral inhibition within a receptive field. However, when the peripheral retina $\left(10^{\circ}\right.$ and $20^{\circ}$ from the fovea) was illuminated with the five circular spots, no inhibition could be seen at all, as is shown in the lower diagram of Fig. 3. The concept of the inhibition within a single receptive field cannot account for this finding, because there is no reason to suppose that there is no receptive field in the peripheral retina involved.

It is a well known psychological fact that color contrast can be perceived more easily in the parafoveal region than in the fovea. Recently, Kurosawa and Katayama ${ }^{16)}$ confirmed by the $\zeta$-method that the contrast effect was greatest at $10^{\circ}$ from the fovea. They considered that the phenomenon of retinal induction would be based on lateral neural connections in the retina and that the lateral connections must maximally be developed in the parafoveal regions of the retina. The strongest inhibition observed above in the parafovea may be another functional expression of the supposed maximal lateral connections in this retinal region.

Previously, Schouten and Ornstein ${ }^{10)}$ carried out the same experiment as that illustrated in Fig. 6 by the same binocular method, and concluded that for the glare effect observed by them the neural interaction of the illuminated areas with glare light and test light is more important than the stray light from the glare source.

Boynton and his associates ${ }^{17)}$ measured by a direct physical method, stray light falling on the foveal center as a function of glare angles in the excised human eye. On their stray light-glare angle curve there is no such inflection as has been observed in our $\rho$-glare angle curves. Therefore it is difficult to consider that stray light in the eye causes such an inflection of the $\rho$-glare angle curve. Our data are difficult to interpret in purely physical terms such as stray light, because we have no reason to suppose that scattering of light would be greatest in the parafoveal region where the inhibition is found most conspicuous (Figs. 4, 5, 7 and 8). It is also difficult to interpret from stray light that the cone and the rod systems show quite different behaviours (cf. Table I with Fig. 4 and upper and lower diagrams in Fig. 8). Although our grounds are quite different from those by Schouten and Ornstein, we have reached the same conclusion that the neural interaction is one of the most important factors for the glare effect. 


\section{SUMMARY}

Spatial summation and inhibition were studied with two methods, Motokawa's $\zeta$-method and the binocular brightness match method.

1. The electrical excitability of the eye was measured after exposure to white five spots together and to the central spot of them alone. The excitability was greater for the five spots together (summation), when the distance between the centrally fixated central spot and other four spots was a few degrees in visual angle, but smaller (inhibition) when the distance was $8^{\circ}-11^{\circ}$.

2. Distribution of summation and inhibition in the retina was mapped by exploring each region with 2 spots $3^{\circ}$ apart from one another, and it was found that the central part of about $12^{\circ}$ in diameter was a summation area, which was surrounded by an inhibitory zone of $5^{\circ}$ in width.

3. The rod process which was determined by the $\zeta$-method, showed spatial summation, but no inhibition at all.

4. The effect of a glare patch $\mathrm{G}$ upon the brightness of a test patch $T$ presented to one eye was measured in comparison with a control patch viewed by the other eye, and it was found that the inhibitory effect of $G$ upon $T$ was greatest when $G$ was presented to the inhibitory zone of the retina determined by the $\zeta$-method.

5. Under experimental conditions advantageous for scotopic vision no inhibition could be found by the brightness match method in accordance with the result obtained by the $\zeta$-method.

The author wishes to express his hearty thanks to Prof. K. Motokawa for his kind guidance and encouragement.

\section{References}

1) Piper, H., Zsch. f. Psychol., 1903, 32, 98.

2) Reeves, P., Astrophys. J., 1918, 47, 141.

3) Piéron, H., C. r. Soc. Biol., 1920, 83, 753.

4) Beitel, R.J., J. Gen. Psychol., 1934, 10, 311.

5) Adrian, E. D. \& Matthews, R., J. Physiol., 1926, 63, 378.

6) Adrian, E. D. \& Mathews, R., J. Physiol., 1928, 65, 273.

7) Granit, R., Amer. J. Physiol., 1930, 94, 41.

8) Granit, R. \& Harper, P., Amer. J. Physiol., 1930, 95, 211.

9) Motokawa, K., Tohoku J. Exp. Med., 1949, 51, 179.

10) Schouten, J. F. \& Ornstein, L. S., J. Opt. Soc. Amer., 1939, 29, 168.

11) Motokawa, K., J. Neurophysiol., 1949, 12, 291.

12) Motokawa, K., Ebe, M., Arakawa, Y. \& Oikawa, T., J. Opt. Soc. Amer., 1951, 41, 478.

13) Motokawa, K. \& Ebe, M., Tohoku J. Exp. Med., 1951, 54, 215.

14) Kuffler, S. W., J. Neurophysiol., 1953, 16, 37.

15) Granit, R., Receptors and sensory perception, Yale Univ, Press, London, 1955.

16) Kurosawa, T. \& Katayama, S., Tohoku J. Exp. Med., 1956, 63, 137.

17) Boynton, R. M., Enoch, J. M. \& Bush, W. R., J. Opt. Soc. Amer., 1954, 44, 879. 